\title{
Evaluation of Walking Ability Using Variance Fractal Dimension Trajectory
}

\author{
Guangan Ren, Yasser Aleed, Witold Kinsner, Nariman Sepehri \\ University of Manitoba \\ 75A Chancellors Circle, Winnipeg, Canada \\ reng34@myumanitoba.ca; yasseraleed@gmail.com; witold.kinsner@umanitoba.ca; nariman.sepehri@umanitoba.ca
}

\begin{abstract}
This paper describes a multiscale time-domain technique for evaluation of gait status of patients who are suffering from diseases such as stroke. This technique is based on variance fractal dimension trajectory (VFDT) algorithm that is applied to a shank acceleration signal. The signal is collected via an inertial measurement unit (IMU). However, its sampling frequency is not constant, and therefore interpolation is employed. Next frame size and step size are chosen properly to guarantee that the signals within all frames are stationarity. Next in order to avoid aliasing phenomenon, Nyquist theorem is checked. Finally VFDT is calculated and error is estimated. Results show that paralyzed legs have higher dimension values than healthy ones.
\end{abstract}

Keywords: Fractals, Shank Acceleration, Stationary, Variance Fractal Dimensions Trajectory (VFDT).

\section{Introduction}

Stroke, as one of the most severe diseases, could cause patients chronic disability. With the help of automatic training systems such as robotic exoskeleton, patients could recover partial or even full walking ability. Therefore, it is necessary to monitor and evaluate their gait status continuously in order to make sure that whether further treatments could be employed. However, to carry out such evaluation is always inconvenient because the injured patients have to move between their houses and hospitals. Recently, inertial measurement units (IMUs) are commonly used to solve the problem because they are portable and data could be collected remotely.

After obtaining data, several evaluation methods have been studied on healthy subjects considering walking speed [1, 2], stride frequency [3], human kinematic [4], and age differences [5]. The gait evaluation on paralyzed patients has also been further conducted [5-8].

The features used for classification could be extracted from time domain, frequency domain or time-frequency domain. Frequency features, such as mean frequency, median frequency and power spectrum deformation, are not strong enough to distinguish healthy lags with paralyzed ones because human could walk at variable speed with changing frequency even a person is paralyzed. In time domain, most of features such as statistical parameters are calculated based on single scale. Fractals, on the other hand, employ multi-scale measures and poly-scale analysis, and therefore could extract more useful features for classification. Morphological-based dimensions are suitable if distribution of a measure is uniform because it treats each intersection equally without considering the structure of the object itself. The spectrumbased dimension is calculated in frequency domain and may introduce artifacts due to Fourier transform. The variance fractal dimension (VFD) was introduced by Kinsner [9] as a polyscale measure of the long-range dependencies in a signal. The VFD ignores the signal power while measuring the signal complexity, and is calculated in a stationary frame within collected data. By shifting the frame along the recorded data, a trajectory of VFD (VFDT) can be obtained.

The VFDT algorithm has been applied to various fields for classification. For example, Kinsner and Grieder [10, 11] used the VFDT to detect external boundaries and internal pauses in speech, and identify internal phonemes of an utterance. Jiao et al. [12] used this technique to distinguish various seismic refraction signals from noise. Barry and Kinsner [13] applied the VFDT for classification of telecommunications traffic.

This paper calculates VFDT of a shank acceleration signal to evaluate human walking ability. The remainder of this paper is organized as follows. Section II tests the signal stationarity. Section III examines Nyquist theorem. Section IV calculates VFDT. Conclusions are provided in Section V. 


\section{Stationary Test Using Moving Window Technique}

As shown in Fig. 1, the shank acceleration is collected by an IMU. And the sampling interval of the signal is shown in Fig. 2. It is seen that the internal is changing from 0.013 to 0.5 seconds. Because cubic spline interpolation could produce the smoothest results and has been applied to many cases $[14,15]$, it is implemented to get a constant sampling frequency $\left(\mathrm{f}_{\mathrm{s}}=10\right.$ kilo samples per second, $\left.\mathrm{kSps}\right)$. The interpolated signal is shown in Fig. 3 . It includes many processes such as background noise (region A), healthy walking (region B) and paralyzed walking (region C). And their details are shown in Fig. 4.

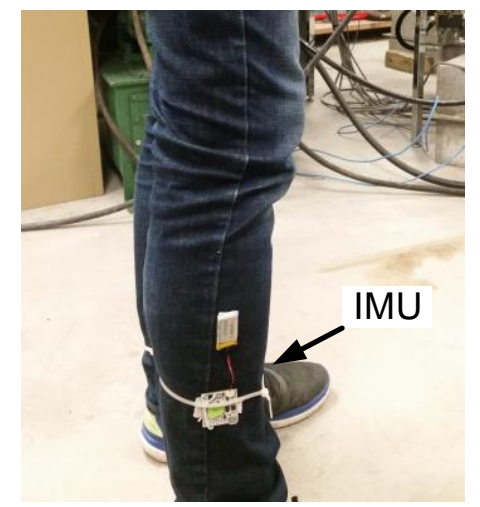

Fig. 1: Typical photo of measuring shank acceleration using IMU.

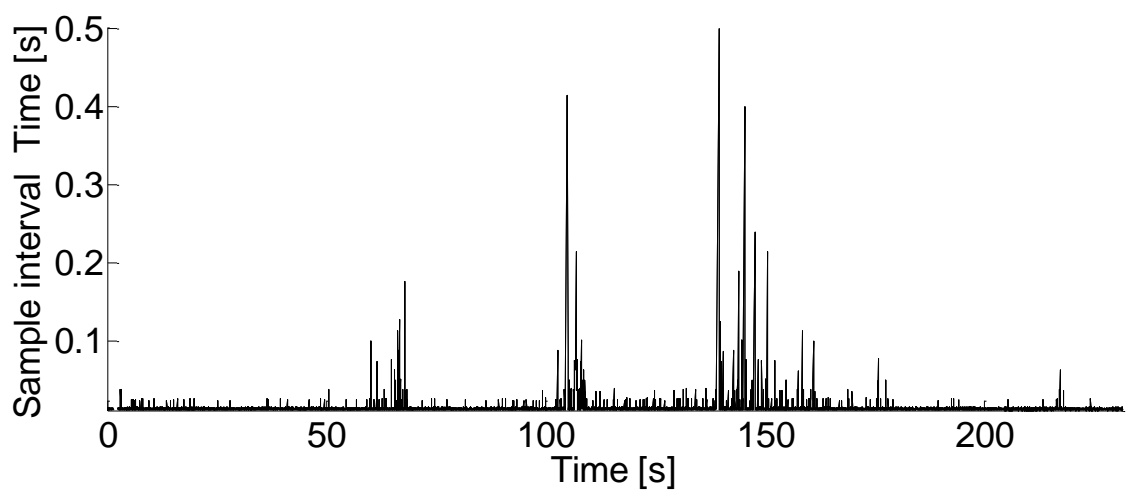

Fig. 2: Sampling interval of collected acceleration signal.

Before analyzing the signal, stationarity test must be implemented. Stationary signals are referred to those whose statistics do not change with time. Especially, the signal is considered to be wide-sense stationary if its mean and autocorrelation are constant. The shank acceleration signal is nonstationary because it includes different processes. Therefore it is necessary to segment the whole signal into small stationary frames. 


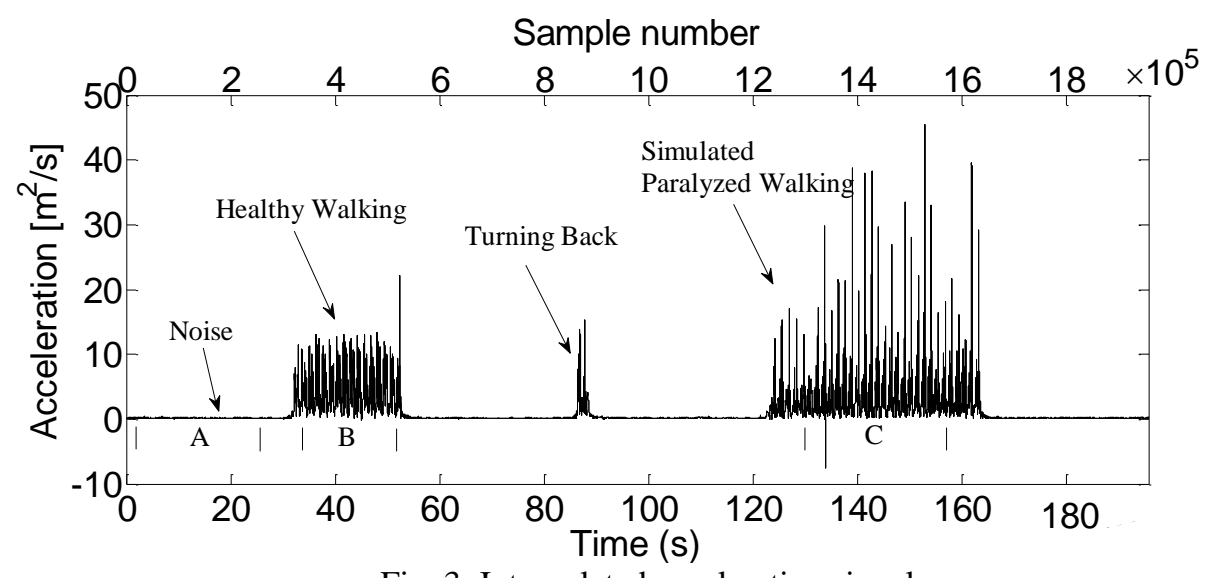

Fig. 3: Interpolated acceleration signal.

In order to guarantee that signal within all moving frames is stationary, frame size and step size must be selected carefully. Large frame size could improve stationarity; however, it takes longer time to detect changes occurring in the signal and the signal within frames is easier to be blurred. So the longer frame size, the weaker local information can be revealed. Too small overlap could cause redundant calculations. Too large overlap causes the loss of detailed information. With frame size of $65536(6.55 \mathrm{~s})$, and overlap=30000 (3 s), for 77\% of frames, the variation in normalized mean is less than $20 \%$ and for $91 \%$ of frames, the variation of autocorrelation for more than $80 \%$ of lags is less than 0.4 . Therefore the process is stationary with about $80 \%$ confidence interval.

\section{Nyquist Test}

FFT (Fast Fourier Transform) was implemented on the worst stationary frame, as shown in Fig. 5. It is a broadband signal, and according to Nyquist sampling theorem, sampling rate $f_{s}$ should be at least twice than the highest frequency $f_{h}$. In order to find white noise, a first-order polynomial is used for data fitting from $4 \mathrm{k} \mathrm{samples/second} \mathrm{to} \mathrm{f}_{\mathrm{s}} / 2$. The fitted line has a slope of 0.00001 . Therefore $\mathrm{f}_{\mathrm{h}}=4 \mathrm{kSps}$ and Nyquist criterion is satisfied. It means than spectral replications are separated and spectral overlapping is avoided.

\section{Variance Fractal Dimension Trajectory}

In order to evaluate gait status, VFD should be calculated on each stationary frame. Therefore a trajectory of VFD (VFDT) is formed.

\subsection{Calculation of VFD for a Frame}

Details of calculating VFD for a frame is provided somewhere else [9-13]. Signal increment for $j^{\text {th }}$ vel at $k^{\text {th }}$ step is

$$
\Delta B_{j k}=B\left(\mathrm{t}_{2 j k}\right)-B\left(\mathrm{t}_{1 j k}\right)
$$

where $B\left(t_{2 j k}\right)$ and $B\left(t_{1 j k}\right)$ are the magnitudes of selected data points at time $t_{2}$ and $t_{1}$ for $j^{\text {th }}$ vel at $k^{\text {th }}$ step respectively. These selected points constitute a vector, and the distance between adjacent data is $\Delta \mathrm{t}_{\mathrm{k}}$, which is the vel size at $k^{\text {th }}$ step and equal to $\mathrm{t}_{2 j k}-\mathrm{t}_{1 j k}$. The variance of all $\Delta \mathrm{B}_{j k}$ at $k^{\text {th }}$ step is

$$
\operatorname{Var}(\Delta B)_{k}=\frac{1}{N_{k}-1}\left[\sum_{j=1}^{N_{k}} \Delta B_{j k}^{2}-\frac{1}{N_{k}}\left(\sum_{j=1}^{N_{k}} \Delta B_{j k}\right)^{2}\right]
$$

In (2), $N_{k}$ is the number of vels at $k^{\text {th }}$ step. When $\Delta \mathrm{t}_{k} \geq 2$, in order to use all data points, it is necessary to use the other vectors by shifting the elements of the above vector by $1,2,3 \ldots \Delta \mathrm{t}_{k}-1$ and calculate the variance of each vector according 
to (2). The average valve of these variances will therefore be

$$
\operatorname{Var}(\Delta B)_{k \text {-average }}=\frac{1}{\Delta t_{k}} \sum_{1}^{\Delta t_{k}} \operatorname{Var}(\Delta B)_{k}
$$

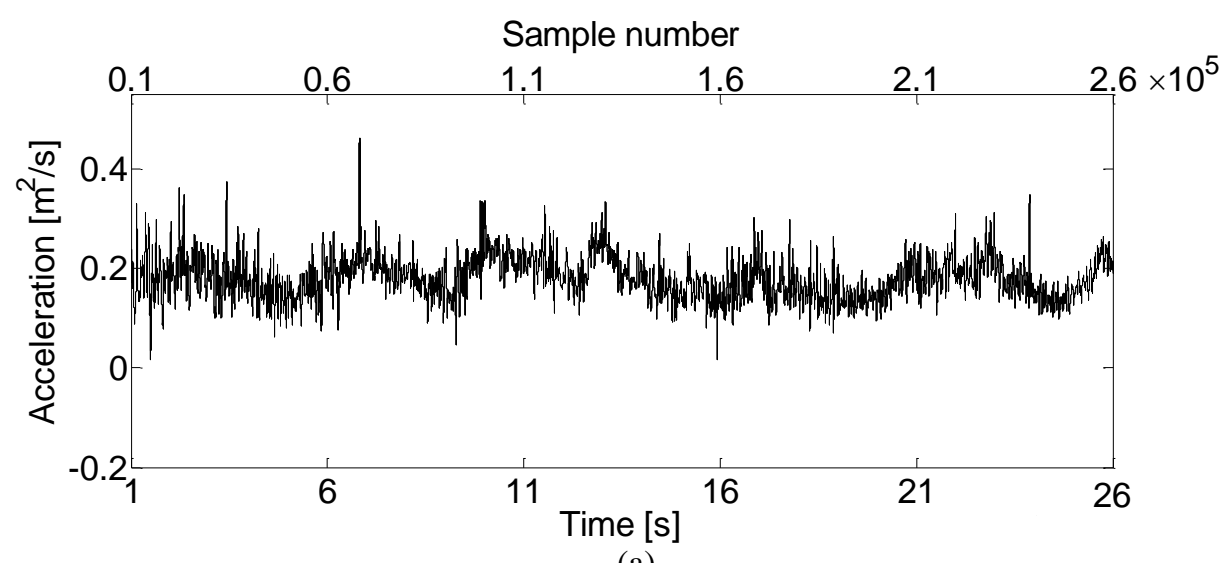

(a)

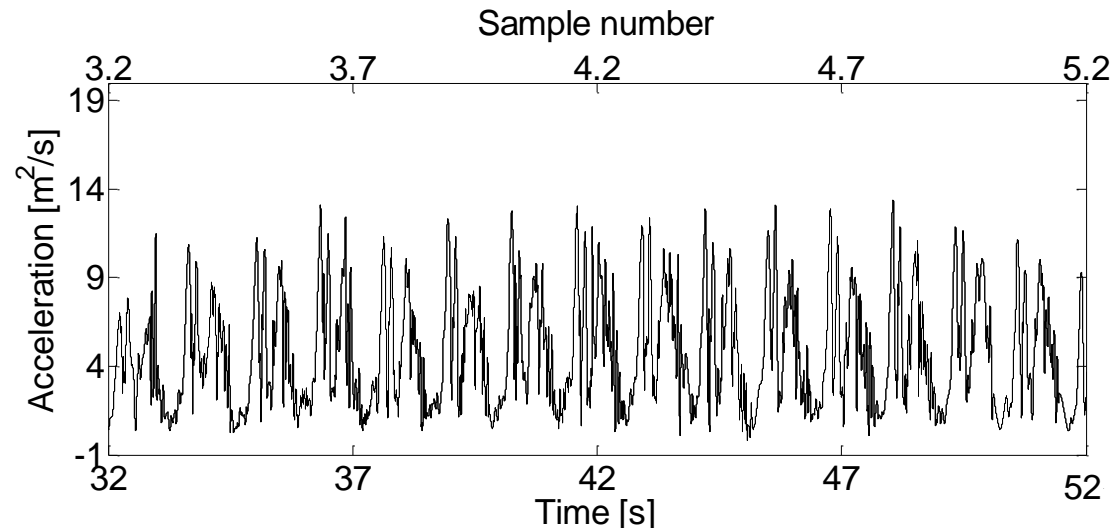

(b)

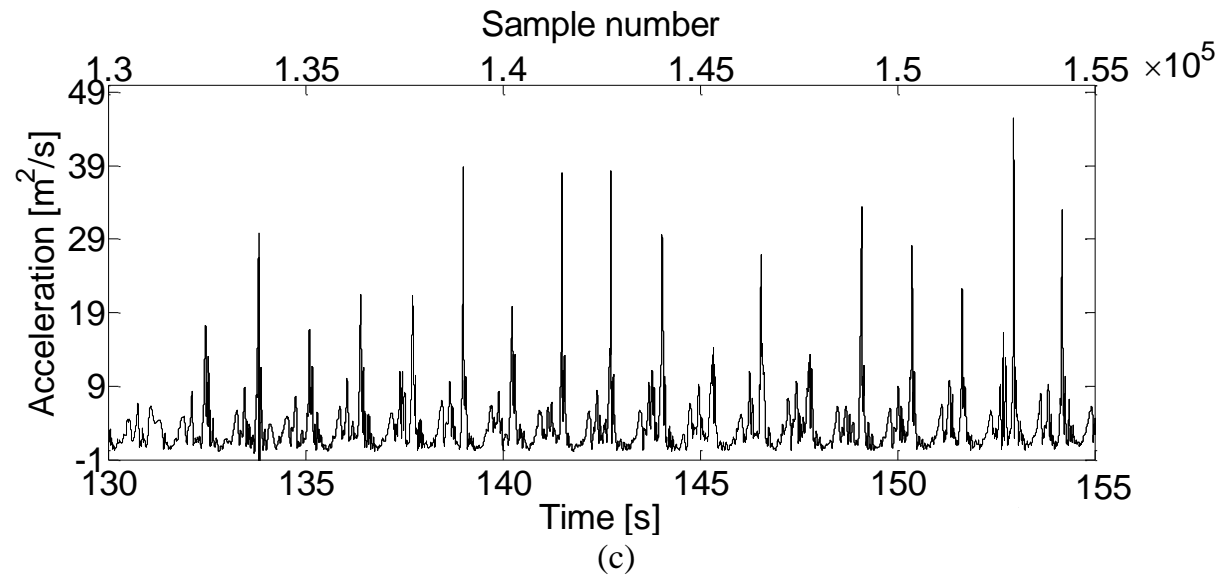

Fig. 4: Details of Fig. 3. (a) Region A: standing still (noise signal). (b) Region B: healthy walking. (c) Region C: simulated paralyzed walking. 


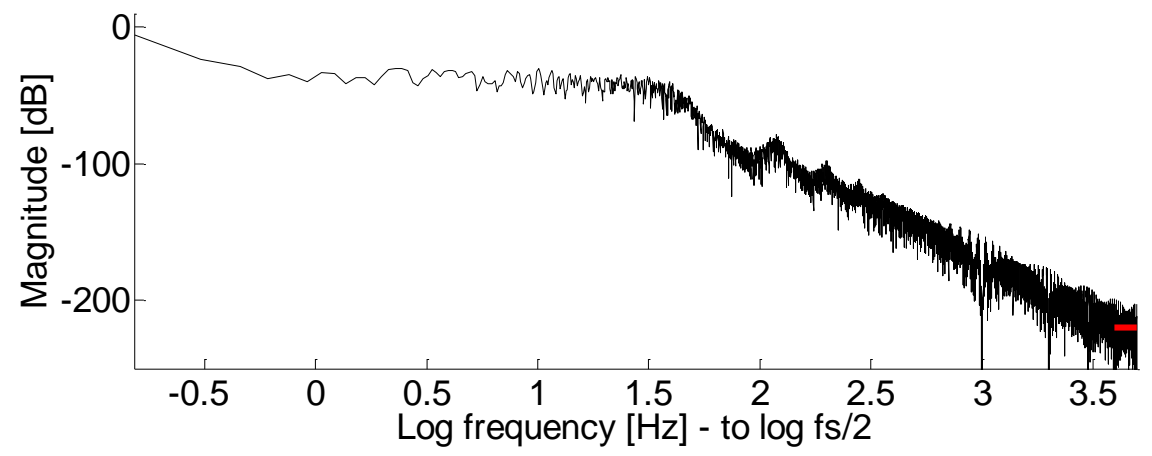

Fig. 5: Power spectral density of worst stationary in Fig. 3.

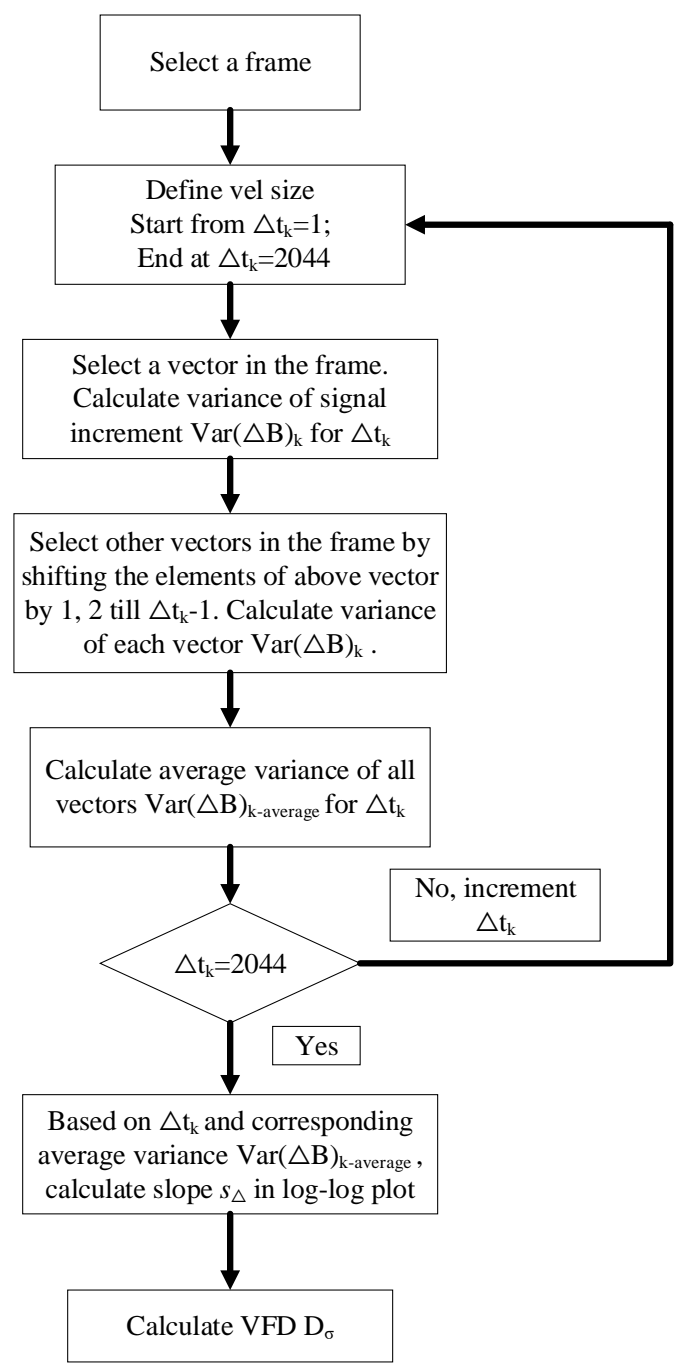

Fig. 6: Flow chart to calculate VFDT.

Then the slope of the average variance $\operatorname{Var}(\Delta \mathrm{B})_{\mathrm{k} \text {-average }}$ verses vel size $\Delta \mathrm{t}_{k}$ in a $\log$-log scale is obtained as follows 


$$
s_{\Delta}=\frac{k \sum_{i=1}^{k} X_{i} Y_{i}-\sum_{i=1}^{k} X_{i} \sum_{i=1}^{k} Y_{i}}{k \sum_{i=1}^{k} X_{i}^{2}-\left(\sum_{i=1}^{k} X_{i}\right)^{2}}
$$

where

$$
X_{k}=\log \left(\Delta t_{k}\right) ; Y_{k}=\log \left[\operatorname{Var}(\Delta \mathrm{B})_{k-\text { average }}\right]
$$

Variance fractal dimension is therefore defined as

$$
D_{\sigma}=2-H
$$

where $H$ is Hurst exponent,

$$
H=\frac{1}{2} s_{\Delta}
$$

In this paper, $\Delta \mathrm{t}_{\mathrm{k}}=1,2,3,4,6,8,10,12,16,20,24,28$ until 2044. The flow chart to calculate VFD is shown in Fig. 6.

\subsection{Calculation of VFDT for All Frames}

Implementing the above algorithm to each frame of the acceleration signal shown in Fig. 3, VFDT could be obtained and is shown in Fig. 7. It is obvious that healthy legs have low dimension value than paralyzed ones, while the background noise has the highest value. This is because VFD is low for a correlated signal, whereas it maintains high value for uncorrelated noise.

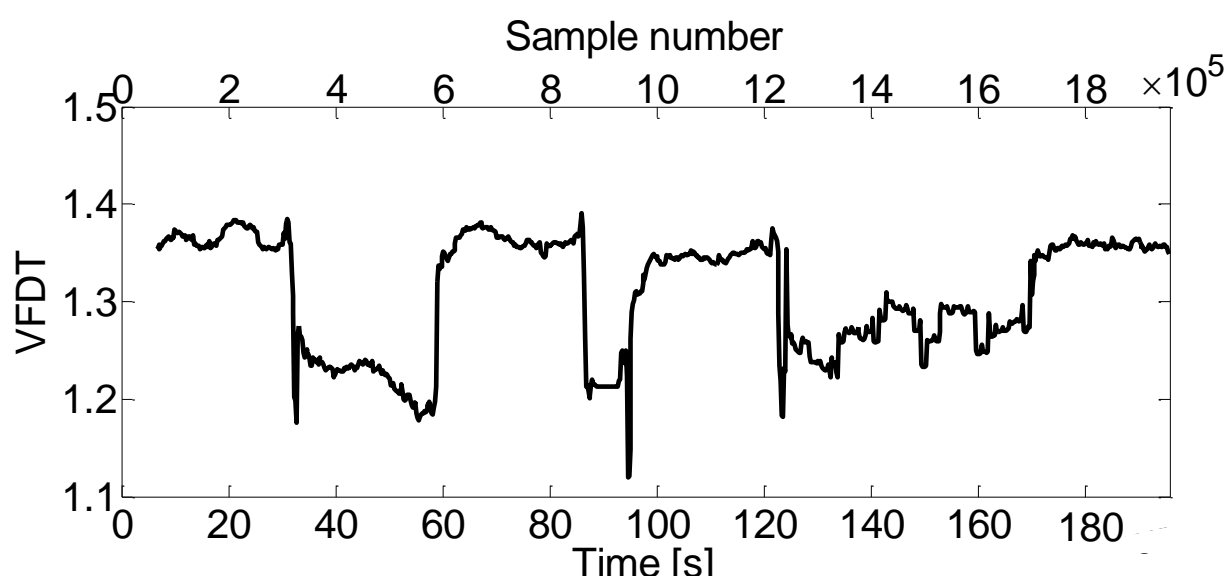

Fig. 7: VFDT of the shank acceleration signal.

The RMS error between all $\operatorname{Var}(\Delta \mathrm{B})_{\mathrm{k} \text {-average }}$ and estimated slope for all the frames is shown in Fig. 8. For $99 \%$ of frames, RMS is less than 0.45. The average variances at different scales and estimated slope of a selected frame whose RMS error is 0.45 are shown in Fig. 9. From Fig. 9, $\operatorname{Var}(\Delta \mathrm{B})_{\mathrm{k} \text {-average }}$ exhibits multifractal because interpolation was employed on the original data. 


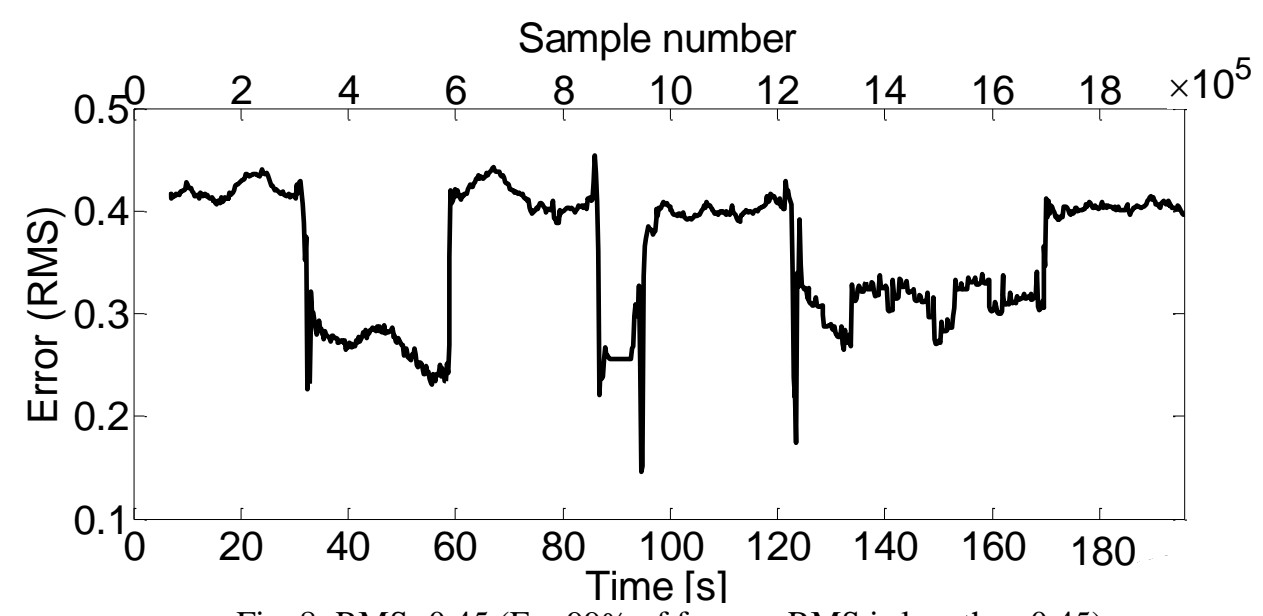

Fig. 8: RMS=0.45 (For 99\% of frames, RMS is less than 0.45).

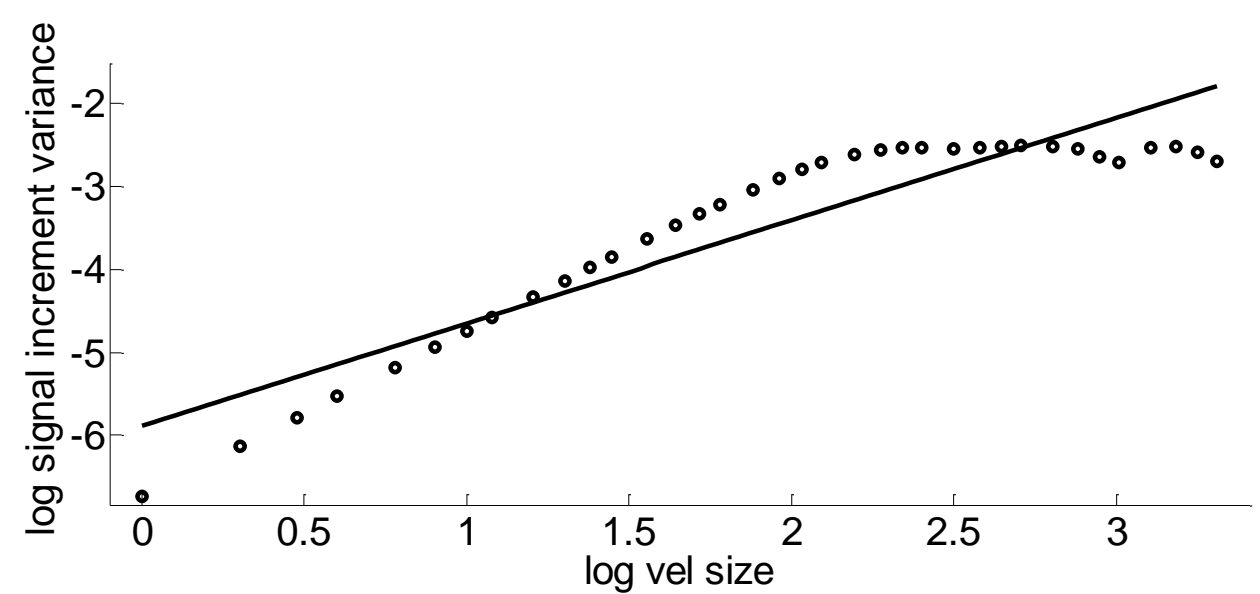

Fig. 9: Average variances at different scales and estimated slope of a selected frame whose RMS error is 0.45 .

\section{Conclusions}

A VFDT of a shank acceleration signal obtained from IMUs was calculated to monitor and evaluate leg conditions. Because VFD had to be calculated on stationary frames, frame size and step size must be selected properly, considering stationary levels, rapidity to detect signal changes, signal blurring, information integrity and calculation speed. In order to use all collected data points, averaging variances of signal increment at different scales was employed. This technique could produce reliable and repeatable results, because the variation in the variances is large.

Our results show that the VFD of injured legs was high because the acceleration signal was not strongly correlated.

\section{References}

[1] M. Q. Liu, F. C. Anderson, M. H. Schwartz, and S. L. Delp, "Muscle contributions to support and progression over a range of walking speeds," J. Biomechanics, vol. 41, pp. 3243-3252, Nov. 2008.

[2] A. R. Den Otter, A. C. H. Geurts, T. Mulder, and J. Duysens, "Speed related changes in muscle activity from normal to very slow walking speeds, " Gait \& Posture, vol. 19, pp. 270-278, Jun. 2004.

[3] T. Klarner, H. K. Chan, J. M. Wakeling, and T. Lam, "Patterns of muscle coordination vary with stride frequency during weight assisted treadmill walking," Gait \& Posture, vol. 31, pp. 360-365, Mar. 2010.

[4] H. Lim, K. Hoon, Y. Soh, A. Tow, and K. Low, "Gait planning for effective rehabilitation-from gait study to application in clinical rehabilitation," in Proc. IEEE 11th ICRR, Kyoto, Japan, 2009. 
[5] A. Schmitz, A. Silder, B. Heiderscheit, J. Mahoney, and D. G. Thelen, "Differences in lower-extremity muscular activation during walking between healthy older and young adults, " J. Electromyography and Kinesiology, vol. 19, pp. 1085-1091, Dec 2009.

[6] M. Iosa, T. Marro, S. Paolucci, and D. Morelli, "Stability and harmony of gait in children with cerebral palsy," Research in Developmental Disabilities, vol. 33, pp. 129-135, Jan-Feb 2012.

[7] S. M. Bruijn, P. Meyns, I. Jonkers, D. Kaat, and J. Duysens, "Control of angular momentum during walking in children with cerebral palsy," Research in Developmental Disabilities, vol. 32, pp. 2860-2866, Nov-Dec 2011.

[8] P. Caliandro, M. Ferrarin, M. Cioni, A. R. Bentivoglio, I. Minciotti, P. I. D'Urso, et al., "Levodopa effect on electromyographic activation patterns of tibialis anterior muscle during walking in Parkinson's disease," Gait \& Posture, vol. 33, pp. 436-441, Mar 2011.

[9] W. Kinsner, "A unified approach to fractal dimensions," Intern. J. Cognitive Informatics and Natural Intelligence, vol. 1, no. 4, pp. 26-46, Oct-Dec 2007.

[10] W. Kinsner and W. Grieder, "Amplification of signal features using variance fractal dimension trajectory," in Proc. IEEE ICCI, Jun. 2009, pp. 201-209.

[11] W. Kinsner and W. Grieder, "Speech segmentation using multifractal measures and amplification of signal features," in Proc. IEEE ICCI, Aug. 2008, pp. 351-357.

[12] L. Jiao, W. Moon, and W. Kinsner, "Variance fractal dimension analysis of seismic refraction signals," in Proc. IEEE WESCANEX: Commun., Power, Computing, May 1997, pp. 163-167.

[13] R. Barry and W. Kinsner, "Multifractal characterization for classification of telecommunications traffic," in Proc. IEEE CCECE, May 2002, pp. 1538-1544.

[14] H. Yan, Z. Peng, K. Cui, and L. Zhang, "Acoustic travel-time measurement in acoustic temperature field monitoring," in Proc. IEEE WCICA, June 2008, pp. 4947-4951.

[15] M. S. Goodwin, S. S. Intille, F. Albinali, and W. F. Velicer, "Automated detection of stereotypical motor movements," J. autism developmental disorders, vol. 41, no. 6, pp. 770-782, 2011. 